PROCEEDINGS OF THE

AMERICAN MATHEMATICAL SOCIETY

Volume 138, Number 7, July 2010, Pages 2321-2333

S 0002-9939(10)10354-2

Article electronically published on March 10, 2010

\title{
HIGHER ORDER SHUFFLE REGULARIZATION FOR MULTIPLE ZETA VALUES
}

\author{
ZHONG-HUA LI
}

(Communicated by Ken Ono)

\begin{abstract}
We study the higher order shuffle regularization for multiple zeta values and define higher order regularized shuffle relations. We find that higher order regularized shuffle relations can be deduced from the group-like property of the Drinfel'd associator.
\end{abstract}

\section{InTRODUCTION AND STATEMENTS OF RESULTS}

Letting $\mathbf{k}=\left(k_{1}, k_{2}, \ldots, k_{n}\right)$ be a sequence of positive integers, the multiple polylogarithm (with one variable) $\mathrm{Li}_{\mathbf{k}}(z)$ is defined by

$$
\begin{aligned}
\operatorname{Li}_{\mathbf{k}}(z) & =\sum_{m_{1}>m_{2}>\cdots>m_{n}>0} \frac{z^{m_{1}}}{m_{1}^{k_{1}} m_{2}^{k_{2}} \cdots m_{n}^{k_{n}}} \\
& =\int_{0}^{z} \underbrace{\frac{d t}{t} \cdots \frac{d t}{t}}_{k_{1}-1} \frac{d t}{1-t} \underbrace{\frac{d t}{t} \cdots \frac{d t}{t}}_{k_{2}-1} \frac{d t}{1-t} \cdots \underbrace{\frac{d t}{t} \cdots \frac{d t}{t}}_{k_{n}-1} \frac{d t}{1-t},
\end{aligned}
$$

where the iterated integral is given by

$$
\int_{0}^{z} f_{1}(t) d t f_{2}(t) d t \cdots f_{m}(t) d t=\int_{z>t_{1}>\cdots>t_{m}>0} f_{1}\left(t_{1}\right) \cdots f_{m}\left(t_{m}\right) d t_{1} \cdots d t_{m} .
$$

It is obvious that $\operatorname{Li}_{\mathbf{k}}(z)$ is absolutely convergent for $|z|<1$. If $k_{1} \geq 2$ (such a $\mathbf{k}$ is called admissible), then $\operatorname{Li}_{\mathbf{k}}(z)$ converges to the multiple zeta value $\zeta(\mathbf{k})$ when $z$ tends to 1:

$$
\begin{aligned}
\lim _{z \rightarrow 1} \operatorname{Li}_{\mathbf{k}}(z)=\zeta(\mathbf{k}) & =\sum_{m_{1}>m_{2}>\cdots>m_{n}>0} \frac{1}{m_{1}^{k_{1} m_{2}^{k_{2}} \cdots m_{n}^{k_{n}}}} \\
& =\int_{0}^{1} \underbrace{\frac{d t}{t} \cdots \frac{d t}{t}}_{k_{1}-1} \frac{d t}{1-t} \underbrace{\frac{d t}{t} \cdots \frac{d t}{t}}_{k_{2}-1} \frac{d t}{1-t} \cdots \underbrace{\frac{d t}{t} \cdots \frac{d t}{t}}_{k_{n}-1} \frac{d t}{1-t} .
\end{aligned}
$$

Set $\operatorname{wt}(\mathbf{k})=k_{1}+k_{2}+\cdots+k_{n}$, which is called the weight of $\mathbf{k}$.

It is known that there are two ways to write a product of two multiple zeta values as a sum of multiple zeta values: one is called the harmonic shuffle product, which follows from the infinite series expressions; the other one is called the shuffle

Received by the editors September 2, 2009, and, in revised form, November 5, 2009.

2010 Mathematics Subject Classification. Primary 11M32, 33B30.

Key words and phrases. Multiple zeta value, shuffle regularization, multiple polylogarithm.

(C)2010 American Mathematical Society Reverts to public domain 28 years from publication 
product, which is deduced from the iterated integral expressions. Therefore we obtain the so-called (finite) double shuffle relations for multiple zeta values. To find "all" rational linear relations among multiple zeta values, we take the regularized processes, which are called the shuffle regularization and the harmonic shuffle regularization. The shuffle regularization uses multiple polylogarithms. Precisely, for any sequence of positive integers $\mathbf{k}$, there exists one $J>0$ such that

$$
\operatorname{Li}_{\mathbf{k}}(z)=P_{\mathbf{k}}(-\log (1-z))+O\left((1-z) \log ^{J}(1-z)\right), \quad z \rightarrow 1,
$$

where $P_{\mathbf{k}}(T) \in \mathbb{R}[T]$ is called the shuffle regularization. For details, one can refer for example to $[3,6$.

In this note, we study the $O$-part of (1.1): the higher order shuffle regularization. For a sequence $\mathbf{k}=\left(k_{1}, \ldots, k_{n}\right)$ of positive integers and a positive integer $i$, the truncated multiple zeta value $\zeta_{i}(\mathbf{k})$ is defined by

$$
\zeta_{i}(\mathbf{k})=\sum_{i>m_{1}>\cdots>m_{n}>0} \frac{1}{m_{1}^{k_{1}} \cdots m_{n}^{k_{n}}} \in \mathbb{Q} .
$$

By induction on the weight of the index, we obtain the following theorem.

Theorem 1.1. For a sequence of positive integers $\mathbf{k}=(\underbrace{1, \ldots, 1}_{m}, \mathbf{k}^{\prime})$ with $m \geq 0$ and $\mathbf{k}^{\prime}$ admissible (here $\mathbf{k}^{\prime}=\left(k_{1}, \ldots, k_{n}\right)$ with $k_{1} \geq 2$ ), there exist polynomials $P_{\mathbf{k}}^{(i)}(T) \in \mathbb{R}[T]$ such that

$$
\operatorname{Li}_{\mathbf{k}}(z)=\sum_{i=0}^{\infty} P_{\mathbf{k}}^{(i)}(-\log (1-z))(1-z)^{i} .
$$

Here the polynomials $P_{\mathbf{k}}^{(i)}(T)$ satisfy the following properties.

(1) We have $P_{\mathbf{k}}^{(0)}(T)=\sum_{j=0}^{m} P_{\mathbf{k}}^{(0 j)} T^{j}$, where $P_{\mathbf{k}}^{(0 j)}$ has the form

$$
P_{\mathbf{k}}^{(0 j)}=\sum_{\substack{\mathbf{w t}\left(\mathbf{k}^{\prime \prime}\right)=\mathbf{w t}(\mathbf{k})-j \\ \mathbf{k}^{\prime \prime}: \text { admissible }}} \beta_{\mathbf{k}, \mathbf{k}^{\prime \prime}, j} \zeta\left(\mathbf{k}^{\prime \prime}\right)
$$

with $\beta_{\mathbf{k}, \mathbf{k}^{\prime \prime}, j} \in \mathbb{Q}$. In particular, we have $P_{\mathbf{k}}^{(0 m)}=\frac{\zeta\left(\mathbf{k}^{\prime}\right)}{m !}$.

(2) For any $i>0$, we have $P_{\mathbf{k}}^{(i)}(T)=\sum_{j=0}^{n} P_{\mathbf{k}}^{(i j)} T^{j}$, where $P_{\mathbf{k}}^{(i j)}$ has the form

$$
\begin{aligned}
& P_{\mathbf{k}}^{(i j)}=\sum_{\substack{a+\mathbf{w t}\left(\mathbf{k}^{(1)}\right)+\mathbf{w t}\left(\mathbf{k}^{(2)}\right)=\mathbf{w t}(\mathbf{k})-j \\
a>0, \mathbf{k}^{(2)}: \text { admissible }}} \alpha_{\mathbf{k}, \mathbf{k}^{(1)}, \mathbf{k}^{(2)}, a, j} \frac{1}{i^{a}} \zeta_{i}\left(\mathbf{k}^{(1)}\right) \zeta\left(\mathbf{k}^{(2)}\right) \\
& \text { with } \alpha_{\mathbf{k}, \mathbf{k}^{(1)}, \mathbf{k}^{(2)}, a, j} \in \mathbb{Q} \text {. }
\end{aligned}
$$

Moreover, the right hand side of (1.2) is absolutely convergent in the domain $\{z \in$ $\mathbb{C}|| 1-z \mid<\varepsilon(\mathbf{k}), \Re(1-z)>0\}$ for some $\varepsilon(\mathbf{k})>0$.

Remark. The rational coefficients $\beta_{\mathbf{k}, \mathbf{k}^{\prime \prime}, j}$ and $\alpha_{\mathbf{k}, \mathbf{k}^{(1)}, \mathbf{k}^{(2)}, a, j}$ are determined by the recursion relations (2.3)-(2.6), and the polynomial $P_{\mathbf{k}}^{(0)}(T)$ is just $P_{\mathbf{k}}(T)$ appearing in (1.1). 
We can obtain some relations for multiple zeta values from the above theorem. Let $\mathbf{k}, \mathbf{k}^{\prime}$ be two sequences of positive integers. Using the shuffle product of iterated integrals, we have that there exist $c_{\mathbf{k}, \mathbf{k}^{\prime}}^{\mathbf{k}^{\prime \prime}} \in \mathbb{Z}_{>0}$ such that

$$
\operatorname{Li}_{\mathbf{k}}(z) \operatorname{Li}_{\mathbf{k}^{\prime}}(z)=\sum_{\mathbf{k}^{\prime \prime}} c_{\mathbf{k}, \mathbf{k}^{\prime}}^{\mathbf{k}^{\prime \prime}} \operatorname{Li}_{\mathbf{k}^{\prime \prime}}(z)
$$

Let

$$
\operatorname{Li}_{\mathbf{k}}(z, T)=\sum_{i=0}^{\infty} P_{\mathbf{k}}^{(i)}(T)(1-z)^{i}
$$

be a formal series. From the shuffle product of multiple polylogarithms and the monodromy of logarithms, we have the following theorem.

Theorem 1.2. For two sequences of positive integers $\mathbf{k}$ and $\mathbf{k}^{\prime}$, we have

$$
\operatorname{Li}_{\mathbf{k}}(z, T) \operatorname{Li}_{\mathbf{k}^{\prime}}(z, T)=\sum_{\mathbf{k}^{\prime \prime}} c_{\mathbf{k}, \mathbf{k}^{\prime}}^{\mathbf{k}^{\prime \prime}} \operatorname{Li}_{\mathbf{k}^{\prime \prime}}(z, T),
$$

where $c_{\mathbf{k}, \mathbf{k}^{\prime}}^{\mathbf{k}^{\prime \prime}}$ is defined as in (1.5).

The proofs of the above two theorems will be given in Section 2 .

By comparing the coefficients of powers of $1-z$ and $T$ in (1.7), we obtain relations of multiple zeta values. We call these relations higher order regularized shuffle relations for multiple zeta values. We list some computations. For example we have

$$
\begin{aligned}
& \operatorname{Li}_{2}(z)=\zeta(2)-\sum_{i=1}^{\infty}\left\{\frac{1}{i}(-\log (1-z))+\frac{1}{i^{2}}\right\}(1-z)^{i} \\
& \operatorname{Li}_{2,2}(z)=\zeta(2,2)-\sum_{i=1}^{\infty}\left\{\left(\frac{1}{i} \zeta(2)+\frac{1}{i} \zeta_{i}(2)\right)(-\log (1-z))\right. \\
&\left.\quad+\frac{1}{i^{2}} \zeta(2)+\frac{1}{i^{2}} \zeta_{i}(2)-\frac{2}{i} \zeta(3)+\frac{2}{i} \zeta_{i}(3)\right\}(1-z)^{i}, \\
& \operatorname{Li}_{3,1}(z)=\zeta(3,1)+\sum_{i=1}^{\infty}\left\{\frac{1}{2 i} \zeta_{i}(1)(-\log (1-z))^{2}+\left(\frac{1}{i^{2}} \zeta_{i}(1)+\frac{1}{i} \zeta_{i}(2)\right)(-\log (1-z))\right. \\
&\left.\quad+\frac{1}{i^{3}} \zeta_{i}(1)+\frac{1}{i^{2}} \zeta_{i}(2)+\frac{1}{i} \zeta_{i}(3)-\frac{1}{i} \zeta(2,1)\right\}(1-z)^{i} .
\end{aligned}
$$

Thus the shuffle product

$$
\operatorname{Li}_{2}(z) \operatorname{Li}_{2}(z)=2 \operatorname{Li}_{2,2}(z)+4 \operatorname{Li}_{3,1}(z)
$$

yields the shuffle relation

$$
\zeta(2) \zeta(2)=2 \zeta(2,2)+4 \zeta(3,1)
$$

and the Euler relation

$$
\zeta(3)=\zeta(2,1) .
$$

Let $A=\left\{e_{0}, e_{1}\right\}$ with $e_{0}$ and $e_{1}$ two noncommutative variables, and let $\mathbb{C}\langle\langle A\rangle\rangle$ be the free associative algebra generated by $e_{0}$ and $e_{1}$. It is known that the Drinfel'd associator $\Phi_{D R}\left(e_{0}, e_{1}\right)$ is a generating function of multiple zeta values (see [2]). In fact, for an admissible $\mathbf{k}=\left(k_{1}, \ldots, k_{n}\right)$, we have

$$
\zeta(\mathbf{k})=(-1)^{n} \times\left(\text { coefficient of } e_{0}^{k_{1}-1} e_{1} \cdots e_{0}^{k_{n}-1} e_{1} \text { in } \Phi_{D R}\right) .
$$


We also find that the higher order regularized shuffle relations for multiple zeta values can be deduced from the group-like property of $\Phi_{D R}$, where the algebra $\mathbb{C}\langle\langle A\rangle\rangle$ has a coproduct $\Delta$ determined by

$$
\Delta\left(e_{i}\right)=e_{i} \otimes 1+1 \otimes e_{i}, \quad i=0,1 .
$$

To state this main result, we define $\operatorname{Shuffle}(\mathbb{C})$ as the set of elements $\Phi\left(e_{0}, e_{1}\right) \in$ $\mathbb{C}\langle\langle A\rangle\rangle$ with the following two properties:

(1) $\Phi\left(e_{0}, 0\right)=\Phi\left(0, e_{1}\right)=1$,

(2) (group-like) $\Delta \Phi=\Phi \otimes \Phi$.

Note that the set of associators (see [1] for the definition of associators) is contained in $\operatorname{Shuffle}(\mathbb{C})$. For an admissible sequence $\mathbf{k}=\left(k_{1}, \ldots, k_{n}\right)$, we define

$$
\zeta_{\Phi}(\mathbf{k})=(-1)^{n} \times\left(\text { coefficient of } e_{0}^{k_{1}-1} e_{1} \cdots e_{0}^{k_{n}-1} e_{1} \text { in } \Phi\right) .
$$

We call the value $\zeta_{\Phi}(\mathbf{k})$ the multiple zeta value associated to $\Phi$. Note that when $\Phi=\Phi_{D R}$, the multiple zeta value $\zeta_{\Phi}(\mathbf{k})$ is nothing but the multiple zeta value $\zeta(\mathbf{k})$. The main theorem of this paper says that these values $\zeta_{\Phi}(\mathbf{k})$ satisfy the same higher order regularized shuffle relations as multiple zeta values.

To state the main theorem, we give the definition of multiple polylogarithms associated to $\Phi\left(e_{0}, e_{1}\right) \in \mathbf{S h u f f l e}(\mathbb{C})$. For a sequence $\mathbf{k}$ of positive integers, we define $\operatorname{Li}_{\mathbf{k}, \Phi}(z, T)$ by replacing the multiple zeta values occurring in (1.6), the defining formula of $\operatorname{Li}_{\mathbf{k}}(z, T)$, with the corresponding multiple zeta values associated to $\Phi$. More specifically, if $\mathbf{k}=(\underbrace{1, \ldots, 1}_{m}, k_{1}, \ldots, k_{n})$ with $m, n \geq 0$ and $k_{1} \geq 2$, we define

$$
\operatorname{Li}_{k, \Phi}(z, T)=\sum_{i=0}^{\infty} P_{\mathbf{k}, \Phi}^{(i)}(T)(1-z)^{i},
$$

where $P_{\mathbf{k}, \Phi}^{(i)}(T) \in \mathbb{C}[T]$ is determined as the following:

- $P_{\mathbf{k}, \Phi}^{(0)}(T)=\sum_{j=0}^{m} P_{\mathbf{k}, \Phi}^{(0 j)} T^{j}$ with

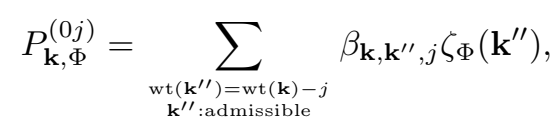

- $P_{\mathbf{k}, \Phi}^{(i)}(T)=\sum_{j=0}^{n} P_{\mathbf{k}, \Phi}^{(i j)} T^{j}$ for any $i>0$ with

$$
P_{\mathbf{k}, \Phi}^{(i j)}=\sum_{\substack{a+\mathbf{w t}\left(\mathbf{k}^{(1)}\right)+\mathbf{w t}\left(\mathbf{k}^{(2)}\right)=\mathrm{wt}(\mathbf{k})-j \\ a>0, \mathbf{k}\left(\mathbf{k}^{(2)}:\right. \text { admissible }}} \alpha_{\mathbf{k}, \mathbf{k}^{(1)}, \mathbf{k}^{(2)}, a, j} \frac{1}{i^{a}} \zeta_{i}\left(\mathbf{k}^{(1)}\right) \zeta_{\Phi}\left(\mathbf{k}^{(2)}\right) .
$$

Now we give the main theorem.

Theorem 1.3. Let $\Phi\left(e_{0}, e_{1}\right) \in \mathbf{S h u f f l e}(\mathbb{C})$ and let $\mathbf{k}, \mathbf{k}^{\prime}$ be two sequences of positive integers. We have

$$
\operatorname{Li}_{\mathbf{k}, \Phi}(z, T) \operatorname{Li}_{\mathbf{k}^{\prime}, \Phi}(z, T)=\sum_{\mathbf{k}^{\prime \prime}} c_{\mathbf{k}, \mathbf{k}^{\prime}}^{\mathbf{k}^{\prime \prime}} \operatorname{Li}_{\mathbf{k}^{\prime \prime}, \Phi}(z, T),
$$

where $c_{\mathbf{k}, \mathbf{k}^{\prime}}^{\mathbf{k}^{\prime \prime}}$ is defined as in (1.5). 
We call the relations of multiple zeta values associated to $\Phi$ deduced from (1.9) the higher order regularized shuffle relations of $\Phi\left(e_{0}, e_{1}\right)$. Obviously, the higher order regularized shuffle relations of $\Phi\left(e_{0}, e_{1}\right)$ contain the shuffle relations of $\Phi$. Some higher order regularized shuffle relations are:

$$
\begin{aligned}
\zeta_{\Phi}(2) \zeta_{\Phi}(2) & =2 \zeta_{\Phi}(2,2)+4 \zeta_{\Phi}(3,1), \\
\zeta_{\Phi}(3) & =\zeta_{\Phi}(2,1) .
\end{aligned}
$$

We will prove the above theorem in Section 3.

\section{Proofs of Theorem 1.1 And Theorem 1.2}

In this section, we prove Theorem 1.1 and Theorem 1.2

Let $\mathbf{k}=(\underbrace{1, \ldots, 1}_{m}, \mathbf{k}^{\prime})$ be a sequence as in Theorem[1.1] We define the polynomials $P_{\mathbf{k}}^{(i)}(T)$ by induction on $\operatorname{wt}(\mathbf{k})$. If $\operatorname{wt}(\mathbf{k})=0$, we set $\operatorname{Li}_{\mathbf{k}}(z)=1$. Now we assume $\mathrm{wt}(\mathbf{k})>0$. There are two cases.

If $m=0$, the sequence $\mathbf{k}=\mathbf{k}^{\prime}=\left(k_{1}, \ldots, k_{n}\right)$ is admissible with $n>0$. Letting $\partial \mathbf{k}=\left(k_{1}-1, k_{2}, \ldots, k_{n}\right)$, we have

$$
\zeta(\mathbf{k})-\operatorname{Li}_{\mathbf{k}}(z)=\int_{z}^{1} \frac{1}{t} \operatorname{Li}_{\partial \mathbf{k}}(t) d t=\sum_{i=1}^{\infty} \int_{z}^{1} \operatorname{Li}_{\partial \mathbf{k}}(t)(1-t)^{i-1} d t
$$

If $m>0$, setting $\mathbf{k}=(1, \partial \mathbf{k})$, we have

$$
\operatorname{Li}_{\mathbf{k}}(z)=\int_{0}^{z} \frac{1}{1-t} \operatorname{Li}_{\partial \mathbf{k}}(t) d t
$$

Using the assumption of induction on $\partial \mathbf{k}$ in (2.1) and (2.2), respectively, we finish the proof of Theorem 1.1. More specifically, we find the recursion relations of $\alpha_{\mathbf{k}, \mathbf{k}^{(1)}, \mathbf{k}^{(2)}, a, j}$ and $\beta_{\mathbf{k}, \mathbf{k}^{\prime \prime}, j}$.

(i) If $m=0$, we have

- for an admissible sequence $\mathbf{k}^{\prime \prime}$ with $\operatorname{wt}\left(\mathbf{k}^{\prime \prime}\right)=\operatorname{wt}(\mathbf{k})$,

$$
\beta_{\mathbf{k}, \mathbf{k}^{\prime \prime}, 0}= \begin{cases}1, & \text { if } \mathbf{k}^{\prime \prime}=\mathbf{k} \\ 0, & \text { otherwise }\end{cases}
$$

- for sequences $\mathbf{k}^{(1)}, \mathbf{k}^{(2)}$ and integers $a, j$ with $a>0,0 \leq j \leq n, a+\operatorname{wt}\left(\mathbf{k}^{(1)}\right)+$ $\operatorname{wt}\left(\mathbf{k}^{(2)}\right)=\operatorname{wt}(\mathbf{k})-j$ and $\mathbf{k}^{(2)}$ admissible,

$$
\alpha_{\mathbf{k}, \mathbf{k}^{(1)}, \mathbf{k}^{(2)}, a, j}= \begin{cases}-\frac{(a+j-1) !}{j !} \alpha_{\partial \mathbf{k}, \mathbf{k}^{\prime \prime}, \mathbf{k}^{(2)}, b, a+j-1}, & \text { if } \mathbf{k}^{(1)}=\left(b, \mathbf{k}^{\prime \prime}\right) \text { with } \\ & b>0, a \leq n+1-j \\ -\frac{(a+j-1) !}{j !} \beta_{\partial \mathbf{k}, \mathbf{k}^{(2)}, a+j-1}, & \text { if } a \leq n+1-j \text { and } \\ 0, & \mathbf{k}^{(1) \text { is empty }} \\ & \text { otherwise. }\end{cases}
$$

(ii) If $m>0$, we have 
- for an admissible sequence $\mathbf{k}^{\prime \prime}$ and an integer $j$ with $0 \leq j \leq m$ and $\operatorname{wt}\left(\mathbf{k}^{\prime \prime}\right)=\operatorname{wt}(\mathbf{k})-j$,

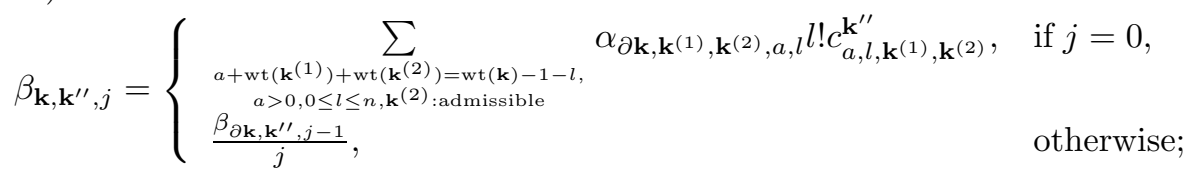

- for sequences $\mathbf{k}^{(1)}, \mathbf{k}^{(2)}$ and integers $a, j$ with $a>0,0 \leq j \leq n, a+\mathrm{wt}\left(\mathbf{k}^{(1)}\right)+$ $\operatorname{wt}\left(\mathbf{k}^{(2)}\right)=\operatorname{wt}(\mathbf{k})-j$ and $\mathbf{k}^{(2)}$ admissible,

$$
\alpha_{\mathbf{k}, \mathbf{k}^{(1)}, \mathbf{k}^{(2)}, a, j}=-\sum_{k=j}^{n} \frac{k !}{j !} \alpha_{\partial \mathbf{k}, \mathbf{k}^{(1)}, \mathbf{k}^{(2)}, a+j-k-1, k} ;
$$

here we assign $\alpha_{\partial \mathbf{k}, \mathbf{k}^{(1)}, \mathbf{k}^{(2)}, a+j-k-1, k}=0$ for $a+j-k-1 \leq 0$.

In the formula (2.5),$c_{a, l, \mathbf{k}^{(1)}, \mathbf{k}^{(2)}}^{\mathbf{k}^{\prime \prime}}$ are integers uniquely determined by the shuffle product

$$
\zeta\left(a+l+1, \mathbf{k}^{(1)}\right) \zeta\left(\mathbf{k}^{(2)}\right)=\sum_{\mathbf{k}^{\prime \prime}: \text { admissible, } \mathrm{wt}\left(\mathbf{k}^{\prime \prime}\right)=\mathrm{wt}(\mathbf{k})} c_{a, l, \mathbf{k}^{(1)}, \mathbf{k}^{(2)}}^{\mathbf{k}^{\prime \prime}} \zeta\left(\mathbf{k}^{\prime \prime}\right) .
$$

We also find that $P_{\mathbf{k}}^{(0)}(T)$ is just the polynomial $P_{\mathbf{k}}(T)$ in (1.1).

To prove Theorem[1.2, we introduce the following lemma.

Lemma 2.1. Let $k \geq 0$ be an integer. Let

$$
f(z)=\sum_{0 \leq n \leq k, m \geq 0} a_{n m}(\log z)^{n} z^{m}
$$

and

$$
g(z)=\sum_{0 \leq n \leq k, m \geq 0} b_{n m}(\log z)^{n} z^{m}
$$

be two absolutely convergent series in the domain $\{z \in \mathbb{C}|| z \mid<\varepsilon, \Re(z)>0\}$ with $a_{n m}, b_{n m} \in \mathbb{C}$, where $\varepsilon>0$ is a small constant. If $f(z)=g(z)$, it follows that

$$
a_{n m}=b_{n m}, \text { for any } 0 \leq n \leq k, m \geq 0 .
$$

Proof. We proceed with the proof by using induction on $k$. The case $k=0$ is trivial. Below we assume that $k>0$.

Let $\rho$ be an operator defined by

$$
\rho(h(z))=h\left(z e^{2 \pi i}\right) .
$$

We have

(a) $(\rho-1)^{m}(\log z)^{n}=\sum_{r=0}^{m}(-1)^{m-r}\left(\begin{array}{c}m \\ r\end{array}\right)(\log z+2 r \pi i)^{n}, m, n \geq 0$.

(b) $(\rho-1)^{m}(\log z)^{n}=0,0 \leq n<m$.

We also define

$$
\log \rho=\log (1+\rho-1):=(\rho-1)-\frac{(\rho-1)^{2}}{2}+\frac{(\rho-1)^{3}}{3}-\cdots+(-1)^{k-1} \frac{(\rho-1)^{k}}{k} .
$$

By (b), the actions of $\log \rho$ on $f(z)$ and $g(z)$ can be regarded as the logarithm of the operator $\rho$. 
Letting

$$
f(z, T)=\sum_{0 \leq n \leq k, m \geq 0} a_{n m} T^{n} z^{m}
$$

we have

$$
e^{2 \pi i \frac{\partial}{\partial T}} f(z, T)=\sum_{0 \leq n \leq k, m \geq 0} a_{n m}(T+2 \pi i)^{n} z^{m}=\rho f(z),
$$

which implies

$$
(\log \rho) f(z)=\left.2 \pi i \frac{\partial}{\partial T} f(z, T)\right|_{T=\log z}=2 \pi i \sum_{0 \leq n \leq k-1, m \geq 0}(n+1) a_{n+1, m}(\log z)^{n} z^{m} .
$$

The condition $f(z)=g(z)$ gives that $(\log \rho) f(z)=(\log \rho) g(z)$. Then by the assumption of induction, we get

$$
a_{n m}=b_{n m}, \quad 1 \leq n \leq k, m \geq 0 .
$$

Thus we can rewrite $f(z)=g(z)$ by

$$
\sum_{m \geq 0} a_{0 m} z^{m}=\sum_{m \geq 0} b_{0 m} z^{m},
$$

which implies that $a_{0 m}=b_{0 m}$ for any $m \geq 0$.

Corollary 2.2. Let $k \geq 0$ be an integer. Let

$$
f(z, T)=\sum_{0 \leq n \leq k, m \geq 0} a_{n m} T^{n} z^{m}
$$

and

$$
g(z, T)=\sum_{0 \leq n \leq k, m \geq 0} b_{n m} T^{n} z^{m}
$$

with $a_{n m}, b_{n m} \in \mathbb{C}$. If $f(z, \log z)=g(z, \log z)$ in the domain $\{z \in \mathbb{C}|| z \mid<$ $\varepsilon, \Re(z)>0\}$ with $\varepsilon>0$ a small constant, we have $f(z, T)=g(z, T)$.

A direct application of Corollary 2.2 induces Theorem 1.2 .

\section{Proof of Theorem 1.3}

This section is devoted to proving Theorem 1.3 We use the language of shuffle algebra and the properties of two special solutions of the Knizhnik-Zamolodchikov equation.

3.1. The Knizhnik-Zamolodchikov equation. We recall some facts about the (formal) Knizhnik-Zamolodchikov (abbreviate to KZ below) equation (see for example [2, 4]). The $\mathrm{KZ}$ equation is a differential equation

$$
\frac{d G}{d z}=\left(\frac{e_{0}}{z}+\frac{e_{1}}{z-1}\right) G(z)
$$

where $e_{0}, e_{1}$ are noncommutative symbols as above, and $G(z)$ is a $\mathbb{C}\left\langle\left\langle e_{0}, e_{1}\right\rangle\right\rangle$-valued analytic function. It is known that there exist unique solutions $G_{0}$ and $G_{1}$ of the $\mathrm{KZ}$ equation with the asymptotic properties

$$
G_{0}(z) \sim z^{e_{0}}(z \rightarrow 0), G_{1}(z) \sim(1-z)^{e_{1}}(z \rightarrow 1) .
$$

The Drinfel'd associator $\Phi_{D R}\left(e_{0}, e_{1}\right) \in \mathbb{C}\left\langle\left\langle e_{0}, e_{1}\right\rangle\right\rangle$ is defined as

$$
G_{0}(z)=G_{1}(z) \Phi_{D R}\left(e_{0}, e_{1}\right) .
$$


3.2. The shuffle algebra and shuffle regularization maps. Let $X=\left\{x_{0}, x_{1}\right\}$ be a set of letters. We denote the set of words on $X$ by $X^{*}$, which contains the empty word 1. Similarly, for $A=\left\{e_{0}, e_{1}\right\}$ introduced in Section 1, the set of words is denoted by $A^{*}$. There is a bijection $\phi: A^{*} \rightarrow X^{*}$ given by $\phi(1)=1$ and $\phi\left(e_{i_{1}} e_{i_{2}} \cdots e_{i_{n}}\right)=x_{i_{1}} x_{i_{2}} \cdots x_{i_{n}}$.

Let $\mathfrak{h}=\mathbb{C}\langle X\rangle$ be the $\mathbb{C}$-algebra of noncommutative polynomials on $x_{0}$ and $x_{1}$. It has two subalgebras:

$$
\mathfrak{h}^{1}=\mathbb{C} 1+\mathfrak{h} x_{1}, \mathfrak{h}^{0}=\mathbb{C} 1+x_{0} \mathfrak{h} x_{1} .
$$

The shuffle product $\amalg$ on $\mathfrak{h}$ is defined by linearity and the axioms

S1: $w \amalg 1=1 \amalg w=w$,

S2: $x_{i} u \amalg x_{j} v=x_{i}\left(u \amalg x_{j} v\right)+x_{j}\left(x_{i} u \amalg v\right)$,

for any $x_{i}, x_{j} \in X$ and $w, u, v \in X^{*}$. Under this new product, $\mathfrak{h}$ becomes a commutative algebra, and $\mathfrak{h}^{1}, \mathfrak{h}^{0}$ are still subalgebras. We use the notation $\mathfrak{h}_{\amalg}, \mathfrak{h}_{\amalg}^{1}$ and $\mathfrak{h}_{\amalg}^{0}$ to emphasize this commutative algebra structure.

By [7], the shuffle algebras $\mathfrak{h}_{\amalg}$ and $\mathfrak{h}_{\amalg}^{1}$ can be decomposed as

$$
\mathfrak{h}_{\amalg}=\bigoplus_{j=0}^{\infty} \mathfrak{h}_{\amalg}^{1} \amalg x_{0}^{\amalg j}=\bigoplus_{i, j=0}^{\infty} x_{1}^{\amalg i} \amalg \mathfrak{h}_{\amalg}^{0} \amalg x_{0}^{\amalg j}
$$

and

$$
\mathfrak{h}_{\amalg}^{1}=\bigoplus_{i=0}^{\infty} x_{1}^{\amalg i} \amalg \mathfrak{h}_{\amalg}^{0},
$$

respectively. We define the shuffle regularization maps $\operatorname{reg}_{\amalg}^{1}: \mathfrak{h}_{\amalg} \rightarrow \mathfrak{h}_{\amalg}^{1}$ and $\operatorname{reg}_{\amalg}: \mathfrak{h}_{\amalg} \rightarrow \mathfrak{h}_{\amalg}^{0}$ as in [3] by taking the constant terms of the above decompositions. More explicitly, for any $w \in \mathfrak{h}$, if

$$
w=\sum_{j \geq 0} w_{j} \amalg x_{0}^{\amalg j}=\sum_{i, j \geq 0} x_{1}^{\amalg i} \amalg w_{i j} \amalg x_{0}^{\amalg j}
$$

with $w_{j} \in \mathfrak{h}^{1}$ and $w_{i j} \in \mathfrak{h}^{0}$, we have

$$
\operatorname{reg}_{\amalg}^{1}(w)=w_{0}, \operatorname{reg}_{\amalg}(w)=w_{00} .
$$

The regularization maps are shuffle algebra homomorphisms. The restriction of $\operatorname{reg}_{\amalg}$ to $\mathfrak{h}_{\amalg}^{1}$ will still be denoted by $\operatorname{reg}_{\amalg}$.

3.3. Representations of $G_{0}(z)$ and $G_{1}(z)$ by multiple polylogarithms. We recall the representations of $G_{0}(z)$ and $G_{1}(z)$ by multiple polylogarithms from [5].

Set $\tilde{\operatorname{Li}}(1 ; z)=1$. For $w=x_{0}^{k_{1}-1} x_{1} \cdots x_{0}^{k_{n}-1} x_{1} \in \mathfrak{h}^{1}$, we define

$$
\widetilde{\mathrm{Li}}(w ; z)=(-1)^{n} \operatorname{Li}_{k_{1}, \ldots, k_{n}}(z) \text {. }
$$

For $w \in \mathfrak{h}^{1}, \tilde{\operatorname{Li}}(w ; z)$ is defined by linearly extending. For any $w \in \mathfrak{h}$, there is a unique expression of $w$ as

$$
w=w_{0}+w_{1} \amalg x_{0}+w_{2} \amalg x_{0}^{\amalg}+\cdots+w_{n} \amalg x_{0}^{\amalg n}
$$

with $w_{0}, w_{1}, \ldots, w_{n} \in \mathfrak{h}^{1}$. Then $\tilde{\operatorname{Li}}(w ; z)$ is defined as

$$
\widetilde{\operatorname{Li}}(w ; z)=\sum_{i=0}^{n} \widetilde{\operatorname{Li}}\left(w_{i} ; z\right)(\log z)^{i} .
$$


The functions $\widetilde{\operatorname{Li}}(w ; z)(w \in \mathfrak{h})$ are called multiple polylogarithms too. They also satisfy shuffle relations and have the desired properties

$$
\frac{d}{d z} \widetilde{\operatorname{Li}}\left(x_{i} w ; z\right)=\frac{1}{z-i} \tilde{\operatorname{Li}}(w ; z),
$$

where $w \in \mathfrak{h}$ and $i=0,1$. Hence by the following lemma and the asymptotic arguments, we have

$$
G_{0}(z)=\sum_{w \in A^{*}} \tilde{\operatorname{Li}}(\phi(w) ; z) w
$$

Here $\phi: A^{*} \rightarrow X^{*}$ is the bijection introduced in the above subsection.

Lemma 3.1. Let $G(z)$ be a $\mathbb{C}\left\langle\left\langle e_{0}, e_{1}\right\rangle\right\rangle$-valued function. If $G(z)$ is written as $G(z)=\sum_{w \in A^{*}} g(\phi(w) ; z) w$, then $G(z)$ is a solution of the $K Z$ equation if and only if

$$
\left\{\begin{array}{l}
\frac{d}{d z} g(1 ; z)=0, \\
\frac{d}{d z} g\left(x_{i} w ; z\right)=\frac{1}{z-i} g(w ; z), \quad \forall w \in X^{*}, i=0,1 .
\end{array}\right.
$$

By using the transformation $z \rightarrow 1-z$, one has

$$
G_{1}(z)=\sum_{w \in A^{*}} \tilde{\operatorname{Li}}(\sigma(\phi(w)) ; 1-z) w,
$$

where $\sigma$ is the automorphism of the algebra $\mathfrak{h}$ determined by $\sigma\left(x_{j}\right)=x_{1-j}$ for $j=0,1$.

By the representations of $G_{0}(z)$ and $G_{1}(z)$ via multiple polylogarithms, and the fact that multiple polylogarithms satisfy shuffle relations, it is easy to see that $G_{0}(z)$ and $G_{1}(z)$ are group-like elements with respect to the coproduct $\Delta$ of $\mathbb{C}\left\langle\left\langle e_{0}, e_{1}\right\rangle\right\rangle$ introduced in Section 1.

3.4. The multiple polylogarithms associated to $\Phi\left(e_{0}, e_{1}\right)$. We come to the proof of Theorem 1.3. Let $\Phi\left(e_{0}, e_{1}\right) \in \operatorname{Shuffle}(\mathbb{C}) \subset \mathbb{C}\langle\langle A\rangle\rangle$. We define a linear $\operatorname{map} \widetilde{Z}_{\Phi}: \mathfrak{h}^{0} \rightarrow \mathbb{C}$ by $\widetilde{Z}_{\Phi}(1)=1$ and

$$
\widetilde{Z}_{\Phi}(w)=(-1)^{n} \zeta_{\Phi}\left(k_{1}, \ldots, k_{n}\right)
$$

for any word $w=x_{0}^{k_{1}-1} x_{1} \cdots x_{0}^{k_{n}-1} x_{1} \in \mathfrak{h}^{0}$ with $k_{1} \geq 2$. Then as in [5], we have

$$
\Phi\left(e_{0}, e_{1}\right)=\sum_{w \in A^{*}} \widetilde{Z}_{\Phi}\left(\operatorname{reg}_{\amalg}(\phi(w))\right) w .
$$

Definition 3.2. For any $w \in \mathfrak{h}$, we define a formal series $\widetilde{\mathrm{Li}_{\Phi}}(w ; z) \in$ $\mathbb{C}[[1-z]][\log (1-z)]$ by the following steps:

(i) Set $\widetilde{\operatorname{Li}}_{\Phi}(1 ; z)=1$.

(ii) For any word $w=x_{1}^{m} x_{0}^{k_{1}-1} x_{1} \cdots x_{0}^{k_{n}-1} x_{1} \in \mathfrak{h}^{1}$ with $m, n \geq 0, m+n>0$ and $k_{1} \geq 2$, we define

$$
\widetilde{\operatorname{Li}}_{\Phi}(w ; z)=(-1)^{n+m} \operatorname{Li}_{\mathbf{k}, \Phi}(z,-\log (1-z)),
$$

where $\mathbf{k}=(\underbrace{1, \ldots, 1}_{m}, k_{1}, \ldots, k_{n})$ and $\operatorname{Li}_{\mathbf{k}, \Phi}(z, T)$ is defined as in (1.8). For any $w \in \mathfrak{h}^{1}, \widetilde{\operatorname{Li}}_{\Phi}(w ; z)$ is defined by linearly extending. We also define $\widetilde{P}_{w, \Phi}^{(i)}(T)$ such that

$$
\widetilde{\mathrm{Li}}_{\Phi}(w ; z)=\sum_{i=0}^{\infty} \widetilde{P}_{w, \Phi}^{(i)}(-\log (1-z))(1-z)^{i} .
$$


(iii) For any $w=\sum_{i=0}^{n} w_{i} \amalg x_{0}^{\amalg i} \in \mathfrak{h}$ with $w_{0}, w_{1}, \ldots, w_{n} \in \mathfrak{h}^{1}$, we define

$$
\widetilde{\operatorname{Li}} \Phi(w ; z)=\sum_{i=0}^{n} \widetilde{\operatorname{Li}_{\Phi}}\left(w_{i} ; z\right)(\log z)^{i}
$$

where $\log z=-\sum_{n=1}^{\infty} \frac{(1-z)^{n}}{n}$.

We call $\widetilde{\operatorname{Li}}_{\Phi}(w ; z)(w \in \mathfrak{h})$ the multiple polylogarithm associated to $\Phi$ too.

Set

$$
G_{0}^{\Phi}(z)=\sum_{w \in A^{*}} \widetilde{\operatorname{Li}}_{\Phi}(\phi(w) ; z) w \in \mathbb{C}[[1-z]][\log (1-z)]\left\langle\left\langle e_{0}, e_{1}\right\rangle\right\rangle .
$$

It is obvious that $G_{0}^{\Phi_{D R}}(z)=G_{0}(z)$. To prove Theorem 1.3, it is enough to show the following theorem.

Theorem 3.3. For any $\Phi\left(e_{0}, e_{1}\right) \in \operatorname{Shuffle}(\mathbb{C}), G_{0}^{\Phi}(z)$ is group-like. In particular, we have

$$
\widetilde{\mathrm{Li}}_{\Phi}\left(w_{1} \amalg w_{2} ; z\right)=\widetilde{\mathrm{Li}}_{\Phi}\left(w_{1} ; z\right) \widetilde{\mathrm{Li}}_{\Phi}\left(w_{2} ; z\right)
$$

for any $w_{1}, w_{2} \in \mathfrak{h}$.

The above theorem is a direct consequence of the following proposition.

Proposition 3.4. For any $\Phi\left(e_{0}, e_{1}\right) \in \operatorname{Shuffle}(\mathbb{C})$, we have

$$
G_{0}^{\Phi}(z) \Phi\left(e_{0}, e_{1}\right)^{-1}=G_{1}(z) .
$$

In the rest of the paper, we prove Proposition 3.4 by using the following two lemmas.

For any $\sum_{i \geq 0} f_{i}(z)(\log (1-z))^{i} \in \mathbb{C}[[1-z]][\log (1-z)]$, we define its formal derivation as

$\frac{d}{d z} \sum_{i \geq 0} f_{i}(z)(\log (1-z))^{i}=\sum_{i \geq 0}\left\{\frac{i}{z-1} f_{i}(z)(\log (1-z))^{i-1}+\frac{d f_{i}(z)}{d z}(\log (1-z))^{i}\right\}$.

So we can compute the usual derivation.

Lemma 3.5. For any $w \in \mathfrak{h}$ and $i=0,1$, we have

$$
\frac{d}{d z} \widetilde{\mathrm{Li}}_{\Phi}\left(x_{i} w ; z\right)=\frac{1}{z-i} \widetilde{\mathrm{Li}}_{\Phi}(w ; z) .
$$

Proof. We first prove that (3.2) holds for any word $w \in \mathfrak{h}^{1}$. When $w=1$, $\widetilde{\mathrm{Li}}_{\Phi}\left(x_{i} w ; z\right)$ is $\log z$ if $i=0$ and $-\log (1-z)$ if $i=1$. So (3.2) is valid for $w=1$. When $w \in \mathfrak{h}^{1}$ is a nonempty word, from the proof of Theorem 1.1 (especially (2.1) and (2.2) $)$ and the definition of multiple polylogarithms associated to $\Phi$, we get

$$
\widetilde{\operatorname{Li}}_{\Phi}\left(x_{0} w ; z\right)=\widetilde{Z}_{\Phi}\left(x_{0} w\right)-\int_{z}^{1} \frac{1}{t} \widetilde{\operatorname{Li}}_{\Phi}(w ; t) d t
$$

and

$$
\widetilde{\operatorname{Li}}_{\Phi}\left(x_{1} w ; z\right)=c_{w}-\int_{0}^{z} \frac{1}{1-t} \widetilde{\operatorname{Li}}_{\Phi}(w ; t) d t,
$$

where $c_{w}$ is a constant number. Hence (3.2) holds for any word $w \in \mathfrak{h}^{1}$ and then for any $w \in \mathfrak{h}^{1}$.

Then as in $[5$, the equality (3.2) holds for all $w \in \mathfrak{h}$. 
For any $\sum_{i \geq 1, j \geq 0} a_{i j}(1-z)^{i}(\log (1-z))^{j} \in \mathbb{C}[[1-z]][\log (1-z)]$ with $a_{i j} \in \mathbb{C}$, we also define its formal limit

$$
\lim _{z \rightarrow 1} \sum_{i \geq 1, j \geq 0} a_{i j}(1-z)^{i}(\log (1-z))^{j}=0 .
$$

Then for two elements $f, g \in \mathbb{C}[[1-z]][\log (1-z)]$, we denote

$$
f \sim g, z \rightarrow 1
$$

if

$$
f-g=\sum_{i \geq 1, j \geq 0} a_{i j}(1-z)^{i}(\log (1-z))^{j} \in \mathbb{C}[[1-z]][\log (1-z)]
$$

for some $a_{i j} \in \mathbb{C}$. Under this definition, as an element of $\mathbb{C}[[1-z]][\log (1-$ $z)]\left\langle\left\langle e_{0}, e_{1}\right\rangle\right\rangle$, the solution $G_{1}(z)=G_{0}(z) \Phi_{D R}\left(e_{0}, e_{1}\right)^{-1}$ of the $\mathrm{KZ}$ equation satisfies

$$
G_{1}(z) \sim(1-z)^{e_{1}}=\sum_{i=0}^{\infty} \frac{(\log (1-z))^{i}}{i !} e_{1}^{i}, z \rightarrow 1 .
$$

Lemma 3.6. There exists a unique element $G(z) \in \mathbb{C}[[1-z]][\log (1-z)]\left\langle\left\langle e_{0}, e_{1}\right\rangle\right\rangle$ such that $G(z)$ is a formal solution of the $K Z$ equation and satisfies

$$
G(z) \sim(1-z)^{e_{1}}, z \rightarrow 1 .
$$

Proof. Since $G_{1}(z)$ is such a formal solution, it is sufficient to show the uniqueness.

Assume that $G(z) \in \mathbb{C}[[1-z]][\log (1-z)]\left\langle\left\langle e_{0}, e_{1}\right\rangle\right\rangle$ is a formal solution of the $\mathrm{KZ}$ equation with

$$
G(z) \sim(1-z)^{e_{1}}, \quad z \rightarrow 1 .
$$

Then $G(z)$ has the form

$$
G(z)=(1-z)^{e_{1}}+\sum_{w \in A^{*}} g(\phi(w) ; z) w
$$

with

$$
g(\phi(w) ; z)=\sum_{i \geq 1, j \geq 0} a_{i j}^{\phi(w)}(1-z)^{i}(\log (1-z))^{j},
$$

where $a_{i j}^{\phi(w)} \in \mathbb{C}$. By Lemma 3.1, we have

$$
\left\{\begin{array}{l}
\frac{d}{d z} \tilde{g}(1 ; z)=0, \\
\frac{d}{d z} \tilde{g}\left(x_{i} w ; z\right)=\frac{1}{z-i} \tilde{g}(w ; z), \quad \forall w \in X^{*}, i=0,1,
\end{array}\right.
$$

where for $w \in X^{*}$, we have

$$
\tilde{g}(w ; z)= \begin{cases}g(w ; z), & \text { if } w \neq x_{1}^{n}, n=0,1,2, \ldots, \\ \frac{(\log (1-z))^{n}}{n !}+g(w ; z), & \text { if } w=x_{1}^{n}, n=0,1,2, \ldots\end{cases}
$$

From this, we get

(a) $\frac{d}{d z} g(1 ; z)=0$,

(b) $\frac{d}{d z} g\left(x_{i} w ; z\right)=\frac{1}{z-i} g(w ; z), w \in X^{*}, w \neq x_{1}^{n}, n=0,1, \ldots$,

(c) $\frac{d}{d z} g\left(x_{1} w ; z\right)=\frac{1}{z-1} g(w ; z), w=x_{1}^{n}, n=0,1, \ldots$,

(d) $\frac{d}{d z} g\left(x_{0} w ; z\right)=\frac{1}{z} g(w ; z)+\frac{(\log (1-z))^{n}}{z n !}, w=x_{1}^{n}, n=0,1, \ldots$ 
Now we have

$$
\frac{d}{d z} g(w ; z)=-\sum_{k, l \geq 0}\left((k+1) a_{k+1, l}^{w}+(l+1) a_{k+1, l+1}^{w}\right)(1-z)^{k}(\log (1-z))^{l} .
$$

Then (a) implies that

$$
(k+1) a_{k+1, l}^{1}+(l+1) a_{k+1, l+1}^{1}=0, \quad \forall k, l \geq 0 .
$$

Since there is some $N>0$ such that $a_{k l}^{1}=0$ for all $k \geq 1$ and $l \geq N$, we get $a_{k l}^{1}=0$ for all $k \geq 1$ and $l \geq 0$, which implies that $g(1 ; z)=0$. Similarly, with the help of the formula $\frac{1}{z}=\sum_{k \geq 0}(1-z)^{k},(\mathrm{~b}),(\mathrm{c})$, and (d) yield that

- $k a_{k l}^{x_{1} w}+(l+1) a_{k, l+1}^{x_{1} w}=a_{k l}^{w}, w \in X^{*}, k \geq 1, l \geq 0$,

- $-(k+1) a_{k+1, l}^{x_{0} w}-(l+1) a_{k+1, l+1}^{x_{0} w}=a_{1 l}^{w}+a_{2 l}^{w}+\cdots+a_{k l}^{w}, w \in X^{*}, w \neq x_{1}^{n}$, $k, l \geq 0$,

- $-(k+1) a_{k+1, l}^{x_{0} x_{1}^{n}}-(l+1) a_{k+1, l+1}^{x_{0} x_{1}^{n}}=a_{1 l}^{x_{1}^{n}}+\cdots+a_{k l}^{x_{1}^{n}}, \quad k, l, n \geq 0, l \neq n$,

- $-(k+1) a_{k+1, n}^{x_{0} x_{1}^{n}}-(n+1) a_{k+1, n+1}^{x_{0} x_{1}^{n}}=a_{1 n}^{x_{1}^{n}}+\cdots+a_{k n}^{x_{1}^{n}}+\frac{1}{n !}, \quad k, n \geq 0$.

Using these recursion relations, the fact that for any $w \in X^{*}$ there is some $N>0$ such that $a_{k l}^{w}=0$ for any $k \geq 1, l \geq N$, and the initial condition $a_{k l}^{1}=0$ for any $k \geq 1, l \geq 0$, we see that $g(w ; z)$ is uniquely determined.

Now we prove Proposition 3.4 By Lemma 3.1 and Lemma $3.5 G_{0}^{\Phi}(z)$ is a formal solution of the $\mathrm{KZ}$ equation, so is $G_{0}^{\Phi}(z) \Phi\left(e_{0}, e_{1}\right)^{-1}$. Then by Lemma 3.6, it is sufficient to show that $G_{0}^{\Phi}(z) \Phi\left(e_{0}, e_{1}\right)^{-1}$ has the same asymptotic behavior as $G_{1}(z)$ when $z \rightarrow 1$.

By the definition of $G_{0}^{\Phi}(z)$, we have

$$
G_{0}^{\Phi}(z)=\left(\sum_{w \in A^{*}} \widetilde{\operatorname{Li}}_{\Phi}\left(\operatorname{reg}_{\amalg}^{1}(\phi(w)) ; z\right) w\right) z^{e_{0}} \sim \sum_{w \in A^{*}} \widetilde{\operatorname{Li}}_{\Phi}\left(\operatorname{reg}_{\amalg}^{1}(\phi(w)) ; z\right) w, z \rightarrow 1,
$$

where

$$
\widetilde{\operatorname{Li}}_{\Phi}\left(\operatorname{reg}_{\amalg I}^{1}(\phi(w)) ; z\right) \sim \widetilde{P}_{\operatorname{reg}_{\text {III }}^{1}(\phi(w)), \Phi}^{(0)}(-\log (1-z)), z \rightarrow 1 .
$$

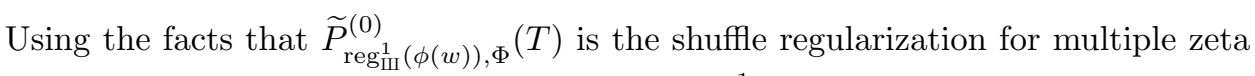
values associated to $\Phi$ as in (1.1) and $\operatorname{reg}_{\amalg} \circ \operatorname{reg}_{\amalg}^{1}=\operatorname{reg}_{\amalg}$, we have

$$
G_{0}^{\Phi}(z) \Phi\left(e_{0}, e_{1}\right)^{-1} \sim(1-z)^{e_{1}}\left(\sum_{w \in A^{*}} \widetilde{Z}_{\Phi}\left(\operatorname{reg}_{\amalg}(\phi(w))\right) w\right) \Phi\left(e_{0}, e_{1}\right)^{-1}, z \rightarrow 1 .
$$

Hence we get $G_{0}^{\Phi}(z) \Phi\left(e_{0}, e_{1}\right)^{-1} \sim(1-z)^{e_{1}}$ when $z \rightarrow 1$.

\section{ACKNOWLEDGEMENTS}

The author would like to express his deep gratitude to Professor Tomohide Terasoma for his constant encouragement, valuable advice and help. The author is grateful to the referee for useful comments. The author was partially supported by the National Natural Science Foundation of China (grant no. 10926111) and the Program for Young Excellent Talents in Tongji University (grant no. 2009KJ065). 


\section{REFERENCES}

[1] P. Deligne and T. Terasoma, Harmonic shuffle relation for associators, preprint, 2005.

[2] V. Drinfel'd, On quasitriangular quasi-Hopf algebras and on a group that is closely connected with Gal $(\overline{\mathbb{Q}} / \mathbb{Q})$ (Russian), Algebra i Analiz 2(1990), no. 4, 149-181; translation in Leningrad Math. J. 2(1991), no. 4, 829-860. MR1080203 (92f:16047)

[3] K. Ihara, M. Kaneko and D. Zagier, Derivation and double shuffle relations for multiple zeta values, Compositio Math. 142 (2006), 307-338. MR2218898 (2007e:11110)

[4] C. Kassel, Quantum groups, Graduate Texts in Mathematics, Vol. 155, Springer-Verlag, New York, 1995. MR.1321145 (96e:17041)

[5] J. Okuda, Duality formulas of the special values of multiple polylogarithms, Bull. London Math. Soc. 37 (2005), 230-242. MR2119023 (2006a:11084)

[6] G. Racinet, Doubles mélanges des polylogarithmes multiples aux racines de l'unité, Publ. Math. Inst. Hautes Études Sci. 95 (2002), 185-231. MR 1953193 (2004c:11117)

[7] C. Reutenauer, Free Lie algebras, Vol. 7 of London Mathematical Society Monographs, The Clarendon Press, Oxford University Press, New York, 1993. MR.1231799 (94j:17002)

Graduate School of Mathematical Science, University of Tokyo, 3-8-1 Komaba, Meguro, TOKYO, 153-8914, JAPAN

Current address: Department of Mathematics, Tongji University, Shanghai 200092, People's Republic of China

E-mail address: lizhmath@gmail.com 